ADVERSE DRUG REACTION

\title{
Clozapine associated dilated cardiomyopathy
}

\author{
M A Tanner, W Culling
}

Postgrad Med J 2003;79:412-413

\begin{abstract}
A 31 year old white man was referred for investigation of a persistent sinus tachycardia. His only significant past medical history was of chronic schizophrenia for which he had been taking clozapine for six years. An electrocardiogram demonstrated sinus tachycardia, voltage criteria for left ventricular hypertrophy, and a prolonged QTC. Echocardiographic findings were consistent with a dilated cardiomyopathy. Serious cardiac complications of clozapine use are rare but have been reported previously. It is important to note that sinus tachycardia may be the only obvious clinical sign, and that complications can manifest months or even years (as in this case) after starting the drug. Patients on clozapine should be informed of potential cardiac symptoms and doctors should maintain a high degree of clinical suspicion throughout the duration of treatment.
\end{abstract}

A 31 year old white man was referred by his general practitioner to the cardiology outpatient department for investigation of a persistent sinus tachycardia. In general he felt well, with no recent history of fever, chest pain, cough, or symptoms suggestive of thyrotoxicosis. On direct questioning there was a history of gradually worsening exertional dyspnoea over several months. His only past medical history of note was chronic schizophrenia which was well controlled on clozapine $400 \mathrm{mg}$ daily. This was prescribed six years previously when flupenthixol had failed to control psychotic symptoms. He was on no other regular medications and did not consume alcohol. He also denied any recreational drug use. His only significant coronary risk factor was a smoking habit of 10 cigarettes daily. There was no family history of either premature coronary disease or cardiomyopathy.

On examination he was afebrile. There was a resting sinus tachycardia of 110 beats/min and blood pressure of 110/75 mm $\mathrm{Hg}$. His jugular venous pressure was raised at $6 \mathrm{~cm}$, but heart sounds were normal with no added sounds or murmurs. The rest of the physical examination was entirely normal.

A resting electrocardiogram (ECG) confirmed sinus tachycardia and changes consistent with left ventricular hypertrophy. The corrected QT interval (QTc) was prolonged at $479 \mathrm{~ms}$ (380-460 ms). Chest radiography demonstrated an enlarged cardiothoracic ratio with clear lung fields.

He proceeded to echocardiography which demonstrated a grossly dilated heart with a left ventricular diastolic dimension of $7.3 \mathrm{~cm}(3.5-5.6 \mathrm{~cm})$. There was very poor global function with an estimated ejection fraction of $9 \%(>50 \%)$. There were no significant valvular abnormalities. Full blood count, urea and electrolytes, liver function tests, ferritin, C-reactive protein, erythrocyte sedimentation rate, thyroid function tests, and troponin I were all normal.

In the absence of any other likely aetiology a presumptive diagnosis of clozapine-induced cardiomyopathy was made and he was admitted for further management. The Committee on Safety of Medicines (CSM) was informed via the yellow card reporting scheme. His clozapine was converted to olanza- pine $10 \mathrm{mg}$, and he was started on a combination of bumetanide, ramipril, and bisoprolol. After seven days there was subjective improvement in his exercise tolerance and there was no clinical evidence of cardiac failure. A predischarge ECG demonstrated a normal QTc interval. He was discharged on the combination of $\beta$-blocker, loop diuretic, and angiotensin converting enzyme inhibitor in addition to his olanzapine.

At his six week follow up appointment there was further subjective improvement. He tolerated his drug regimen well and his mental state remained stable. On examination his sinus tachycardia had resolved and there was no evidence of cardiac failure.

His echocardiogram also demonstrated a significant improvement. Although the left ventricle remained dilated the overall systolic function had improved considerably with an estimated ejection fraction of $43 \%$.

\section{DISCUSSION}

Clozapine is a dibenzodiazepine antipsychotic used in the treatment of resistant schizophrenia. Minor cardiovascular side effects, namely orthostatic hypotension and sinus tachycardia, are relatively common and have been previously described. ${ }^{1}$ There is also a well recognised $1 \%$ risk of agranulocytosis during the first year of treatment. ${ }^{2}$ Of great concern are the lesser reported serious and potentially fatal cardiac complications. In particular both myocarditis and dilated cardiomyopathy have been reported. To date the CSM has received 30 reports of cardiomyopathy and 40 cases of myocarditis associated with clozapine use. Killian et al presented 23 cases of myocarditis and cardiomyopathy temporally associated with this drug. ${ }^{3}$ Between 1989 and 1999 the United States Food and Drug Administration received reports of 28 cases of myocarditis (including 18 deaths) and 41 cases of cardiomyopathy (including 10 deaths) in patients receiving clozapine. ${ }^{4}$ Baysian analysis as applied to the World Health Organisation drug monitoring database has demonstrated that clozapine is significantly more frequently reported in relation to myocarditis and cardiomyopathy than other drugs (including other antipsychotics). Estimation of the incidence of myocarditis in clozapine use ranges between one in 10000 to one in $500 .^{6}$ Interpretation of case reports is complicated by the often non-specific symptoms and signs of myocarditis and cardiomyopathy. ECG changes reported are also non-specific and include sinus tachycardia, T-wave inversion, prolonged QTc, and ST flattening/depression. ${ }^{37}$ It must be borne in mind that many of the cases reported have not had definitive evidence for their diagnoses.

It is, however, very likely that there is a causal link between clozapine and myocarditis/cardiomyopathy. Several cases have been associated with a peripheral and/or cardiac eosinophilia suggestive of an IgE-mediated hypersensitivity reaction. ${ }^{7}$ The greatest risk of cardiac involvement most commonly occurs

Abbreviations: CSM, Committee on Safety of Medicines; ECG, electrocardiogram; QTc, corrected QT interval 


\section{Summary points}

- Cardiac complications of clozapine are rare but potentially fatal.

- Cardiac complications (especially cardiomyopathy) can occur months or even years after the introduction of clozapine treatment.

- Persistent sinus tachycardia may be the sole presenting sign and must not be assumed to be a benign side effect.

- A high degree of clinical suspicion must be maintained in patients on clozapine, and a low threshold for referral for cardiological assessment.

during the first month of use. Although as reported in this case, risk persists throughout its use. ${ }^{7}$ It is also likely that myocarditis typically presents earlier than cardiomyopathy. ${ }^{4}$

In this case there was both subjective and objective evidence of improvement on withdrawal of clozapine. Although it is difficult to know the contribution of drug treatment, there have been reports of improvement on withdrawal of clozapine in both cardiomyopathy ${ }^{8}$ and myocarditis." In addition to standard supportive treatment for myocarditis and cardiomyopathy it has also been suggested that corticosteroids have a role. $^{10}$

In conclusion, clozapine associated myocarditis and cardiomyopathy are serious and potentially lethal complications. Patients should be informed of symptoms of cardiotoxicity. It is important that a high degree of clinical suspicion is maintained throughout the duration of clozapine therapy with a low threshold for referral to a cardiologist.

\section{Authors' affiliations}

M A Tanner, W Culling, Cardiology Department, Kingston Hospital NHS Trust

Correspondence to: Dr Mark Tanner, Cardiology Department, Kingston Hospital, Galsworthy Road, Kingston-upon-Thames, Surrey KT2 7QB, UK; marktanner@lycos.co.uk

Submitted 21 January 2003

Accepted 24 February 2003

\section{REFERENCES}

1 Young CR, Bowers MB, Mazure CM. Management of adverse effects of clozapine. Schizophr Bull 1998;24:381-90.

2 Alvir JM, Lieberman JA, Safferman AZ, et al. Clozapine induced agranulocytosis: incidence and risk factors in the United States. N Engl J Med 1993;329:162-7.

3 Killian JG, Kerr K, Lawrence C, et al. Myocarditis and cardiomyopathy associated with clozapine. Lancet 1999;354:1841-5.

4 La Grenade L, Graham D, Trontell A. Myocarditis and cardiomyopathy associated with clozapine use in the United States. N Engl J Med 2001;345:224-5.

5 Coulter DM, Bate A, Meyboom RHB, et al. Antipsychotic drugs and heart muscle disorder in international pharmacovigilance: data mining study. BM 2001;322:1207-9.

6 Warner B, Alphs L, Schaedelin J, et al. Clozapine and sudden death [letter]. Lancet 2000;355:842.

7 Wooltorton E. Antipsychotic clozapine (Clozaril): myocarditis and cardiovascular toxicity. Canadian Medical Association Journal 2002;166:1185-6.

8 de Kniff DW, Schepers PH, Blanken-Meijs JT. Cardiomyopathy during clozapine therapy. Ned Tijdschr Geneeskd 2001;145:1697-9.

9 Kirpekar VC, Deshpande SM, Joshi PP. Reversible myocarditis in a patient receiving clozapine. Indian Heart J 2001;53:779-81.

10 Hagg S, Spigset O, Bate A, et al. Myocarditis related to clozapine treatment. J Clin Psychopharmacol 2001;21:382-8.

For just US $\$ 25$ you can have instant access to the whole website for 30 days. During this time you will be able to access the full text for all issues (including supplements) available. You will also be able to download and print any relevant pdf files for personal use, and take advantage of all the special features Postgraduate Medical Journal online has to offer.

\section{www.postgradmedj.com}

